\title{
DETERMINING THE VALUE OF SIMULATION IN NURSE EDUCATION: STUDY DESIGN \& INITIAL RESULTS
}

\author{
Corresponding author: \\ Guillaume Alinier, MPhys, PGCE, AMInstP: BHF Project Co-ordinator \\ Hertfordshire Intensive Care \& Emergency Simulation Centre \\ Department of Allied Health Professions \\ University of Hertfordshire \\ College Lane \\ Hatfield \\ Hertfordshire, AL10 9AB \\ Tel: $+44(0) 1707-286395$ \\ Fax: $+44(0) 1707-284199$ \\ Email: G.Alinier@herts.ac.uk
}

William B Hunt, BPharm, PhD, MRPharmS: Associate Dean

Faculty of Health \& Human Sciences

University of Hertfordshire

College Lane

Hatfield

Hertfordshire, AL10 9AB

Tel: $+44(0) 1707-285910$

Fax: $+44(0) 1707-285904$

Email: W.B.Hunt@herts.ac.uk

Ray Gordon, BSc, CEng, MIEE, MIPEM: Associate Head Department of Electronic, Communication and Electrical Engineering University of Hertfordshire

College Lane

Hatfield

Hertfordshire, AL10 9AB

Tel: +44 (0)1707-284187

Fax: +44 (0)1707-284199

Email: R.Gordon@herts.ac.uk 


\section{ABSTRACT}

Nowadays simulation is taking an important place in training and education of healthcare professionals. The University of Hertfordshire is carrying out a study which aims to determine the effect of realistic scenariobased simulation on nursing students' competence and confidence. This project is sponsored by the British Heart Foundation and takes place in the Hertfordshire Intensive Care and Emergency Simulation Centre (HICESC), a simulated three adult beds Intensive Care Unit. The simulation platform used is a Laerdal SimMan Universal Patient Simulator. A unique and robust study design, and results of the study are presented in this article.

Consecutive cohorts of students are being assessed and reassessed after six months using an Objective Structured Clinical Examination (OSCE). Students are randomly divided into a control and experimental group for the period intervening between the two examinations. The experimental group is exposed to simulation training while the other students follow their usual nursing courses. Comparison is made between the OSCE results of the two groups of students. The experimental group had a greater improvement in performance than the control group $(13.43 \%$ compared with $6.76 \%(p<0.05))$. The results and feedback received from students and lecturers suggest that simulation training in nursing education is beneficial.

Keywords: Simulation, OSCE, nursing education, assessment, training. 


\section{INTRODUCTION}

The use of physical three-dimensional simulation to reproduce life-like experiences in order to improve the training of healthcare professionals is developing throughout the world at an unprecedented pace. The general concern for increased patient safety, cost reduction due to human errors, and ethical issues related to training are not unrelated to this phenomenon (Ziv et al. 2000). The Institute of Medicine's 1999 report "To Err is Human: Building a Safer Health System" (Kohn et al. 1999, p.179) encourages all health care organisations and teaching institutions to participate in the development and use of simulation for training novice practitioners. This will probably only occur if there is sufficient scientific evidence to support the proposition that teaching students using very realistic simulation methods justifies the costs that can be involved. It is expected that the most significant cost benefits are indirect and long term (Ziv et al. 2000) but this will be very difficult to judge. The purchase, setup, running and maintenance costs of patient simulators and of the equipment and facilities required can add up to a considerable cost (Issenberg et al. 1999, Lane et al. 2001). Yet the "patient simulator" user community has been growing faster than ever in the last couple of years, and this is being reflected by the creation of national and international simulation societies and associations applied to medicine.

As progress is made in science and technology, the health care equipment and treatment methods are improved. Teaching and training methods need to adapt not only to this progress, but also to the new ethical 
regulations and to the demand for increasing numbers of qualified health care staff. Like many other teaching institutes in the United Kingdom, the University of Hertfordshire prepares hundreds of nurses every year and still needs to increase its training capacity to meet the future National Health Service requirements and yet maintain a good educational standard.

\section{BACKGROUND}

Since 1998, the Hertfordshire Intensive Care and Emergency Simulation Centre (HICESC) has been used to train student nurses and paramedics using low fidelity simulation and part task trainers. Those teaching tools include simple yet very useful models such as full body Advance Life Support trainers, Resusci Annes, intravenous training arms, intubation or airway management heads. Such equipment can be used to improve trainees' practical skills. It has been demonstrated that for some particular skills, such as airway management, they are as effective as using live patients (Roberts

et al. 1997). There are two levels of advanced full body scale simulation, intermediate and high fidelity simulation, which are respectively partly interactive and fully interactive patient simulators or mannequins responding to treatments given. There is no real and scientifically valid evidence which supports the proposition that such equipment and running costs of the simulation facility balance their teaching value and the practical experience they provides to trainees (Ziv et al. 2000). The principle of simulation as a learning and teaching tool draws its ideas from the theories of experiential 
learning (Kolb 1984, Cioffi 2001) and situated learning (Lave \& Wenger 1991). The patient simulation technology that has been developed enables training to take place in a safe and realistic context. Students' participation in learning is expected to help them understand and apply their cognitive and psychomotor skills as they would do in their future professional function. The environment and atmosphere created have to be equivalent to reality to help students suspend disbelief and act as themselves (Streufert et al. 2001, Hegarty \& Bloch 2002).

A number of qualitative and descriptive studies relying on the feedback of candidates exposed to simulation training have been carried out and showed that there was a positive response to the use of simulation as a training tool (Mclndoe 1999, Treadwell \& Grobler 2001, Cleave-Hogg \& Morgan 2002, Murray et al. 2002). It was felt that there was a lack of quantitative research in the overall outcome of students' performance in terms of clinical skills, communication skills and confidence after simulation training (Kneebone 2003), and more particularly in relation to nursing education (Cioffi 2001). The British Heart Foundation has funded a three-year project to investigate how beneficial it is for nursing students to be trained in a simulated specialist ward environment using an intermediate fidelity simulation platform and scenario-based training sessions. The simulation platform used for the study is "SimMan", the Universal Patient Simulator from Laerdal, and is set up in the realistic environment of HICESC, the reproduction of a three adult beds Intensive Care Unit (Alinier et al. 2003). 


\section{STUDY DESIGN}

The different components of the study described below have been granted ethical approval by the Faculty of Health and Human Sciences Research Ethics Committee of the University of Hertfordshire, and informed consent has been obtained from all students involved. The overall design and content have been piloted with a group of nursing students in order to test the different aspects of the study. The validity and authenticity of the simulation scenarios were assessed by a panel of experts from clinical and academic backgrounds. The required amendments were then made to the teaching and assessment methods used. A second and final pilot was conducted to retest the assessment tool. All second year students from the diploma in nursing course are informed of the purpose, the method, and the duration of the study. It is important to note that students are invited to take part in this project on a voluntary basis and are free to withdraw at any time. Although a form of examination is used during the study, it is not linked to any assessment strategy in their course. Students who have fully participated in the study are rewarded with a certificate of attendance to enhance their professional portfolio. By the end of the project it is expected that over 120 students will have taken part and contributed to the better understanding of the use of simulation as a teaching tool. The results of this project will hopefully influence the design of future nursing curricula inside and outside the University of Hertfordshire. 
Figure 1

The study is composed of several phases through which consecutive cohorts of second year diploma nursing students are involved (Figure 1). The students who are involved in the study are separated into a control and experimental group. The different sessions organised are: a "First OSCE session", the "Simulation session", and the "Second OSCE session". In the first instance, only students from the experimental group attend the simulation sessions. The purpose and content of those sessions are explained under the following subheadings.

\section{First OSCE session}

As illustrated in Figure 1, all students are initially tested using an Objective Structured Clinical Examination (OSCE) (Harden \& Gleeson 1979). An OSCE composed of 15 stations has been specifically designed for the study. This first OSCE is used to determine the initial skills level of the students in terms of clinical and communication skills. The OSCE stations address a range of clinical and psychomotor skills (11 stations) and a few cognitive skills (4 stations) as listed in Table 1 . The difficulty level of the stations is such that it is fairly difficult to score $100 \%$ at any of the stations even when students reach the third year of their diploma course and take part in the second OSCE session of the study. Each station is 5 minutes in duration and is followed by a one-minute gap to allow students to rotate to the 
next station. This short break also allows time for the assessors to finish the marking and rearrange the station for the following student. At the start of the session, students are reminded of the aim of the session and given instructions on how it is run. It is important to note that none of the students has had prior experience of an OSCE session. They are told that they may encounter equipment that they have never used before and that they should not worry and simply try to do their best. Examiners have been instructed not to help or give any feedback to the students concerning the different stations at this stage of the study.

\section{Table 1}

\section{$\underline{\text { Simulation session }}$}

After the initial OSCE, which is used to provide an individual baseline measurement, students participating in the study are randomly split into two groups. Half of them are allocated to the control group whereas the other students are allocated to the experimental group. The difference between the two groups is that students from the experimental group are again randomly divided in teams of four students and are required to attend two simulation sessions. Each of those sessions are identical and are organised for two teams of students as illustrated in the session programme (Table 2). All students are equally involved in the initial part of the session. Aspects of teamwork, communication and simulation training are presented and discussed with the students. They are then introduced and exposed to the 
patient simulator before the simulation begins. They are asked to observe the chest rising, feel for the pulse, listen to its chest with a stethoscope and communicate with the mannequin, as it can talk! They are also informed about the type of monitoring equipment that can be used and which procedures can be performed on the patient simulator. It is of utmost importance that students understand what the capabilities of the patient simulator are before the scenario starts. This will greatly affect their experience of participating in the scenarios and influence their behaviour. The whole learning exercise could be jeopardised if students were not adequately briefed and prepared for the simulation.

Only one of the two teams interacts with the patient simulator during any session. On one occasion a team observes the simulation platform from a different area through an audio/video link, and during the other session the team is split into pairs to actively take part in the simulation training while another team of peers observe them. As illustrated in the session plan in Table 2, all students from any one team takes part in two scenarios which are designed to test a range of clinical skills in a ward setting. During these scenarios students are expected to act as "qualified nurses" to care for the patient simulator. When required students can get help from the facilitator who then takes the role of either a doctor or a senior nurse. After having taken part in a scenario, students are debriefed using footage from the video tape recording. Students who were observing the scenarios are invited to participate in the debriefing by sharing their views on aspects such as communication, situation awareness, teamwork, decision making, and clinical 
skills. This immediate feedback given during the debriefing of the students is an important aspect of the simulation session as it is meant to help them reflect positively about their experience with the patient simulator. Since no harm is incurred to a real patient, errors can be allowed to progress so that students can learn from their mistakes without concern of liability or guilt (Ziv et al. 2000). The debriefing can then be used to help students understand their wrongdoing and the most appropriate course of action they should have adopted. It is therefore extremely important that the simulation is followed by debriefing and reflection so that students can learn from the experience (Thiagarajan 1998). Attending two simulation sessions maximises the students' exposure to the simulated environment. They benefit from observing their peers and taking notes, and by taking part in the debriefing of several scenarios.

It is important to note that these sessions are not specifically designed to prepare the students for the second OSCE, but simply provide them with additional clinical experience in a safe and controlled environment. Students might need to use equipment that they encounter during the OSCE, however explanations of how to operate the equipment are not provided. For the OSCE exercises involving technological pieces of equipment, students are asked to use specific functions or settings that they would not need to use during the scenarios.

Table 2 


\section{$\underline{\text { Confidence questionnaire }}$}

At the start of the second OSCE session, students are asked to fill in a questionnaire. It is used to collect the demographic details of the candidates as well as some information concerning their current and past experience in healthcare, their level of confidence, and how stressful they find it working in a technological environment. This information, alongside the OSCE results, will be particularly useful in analysing and explaining their performance or any major differences between the two study groups.

\section{Second OSCE session}

The two OSCE sessions are identical in content and are run as a summative assessment in order to collect the data required for comparison between the two groups. A study by Niehaus et al. (1996) showed that OSCE stations could be effectively repeated after four months without affecting the

results. However, one difference between the first and second OSCE has been incorporated. During the second OSCE, students are given immediate feedback on their performance at each practical station, and this was named "Mixed Mode" OSCE. This process seemed very popular amongst students and teaching staff involved (Alinier 2003). By comparing the results obtained for the first OSCE with those of the second OSCE it is possible to determine whether or not students from the experimental group have improved their skills to a greater extent than those from the control group. To our knowledge 
this is the first study which uses OSCE to quantitatively determine the effectiveness of simulation in nursing education.

For consistency each cohort of students attends the sessions at the same period in their curriculum. The two OSCEs are respectively organised toward the middle of the fourth and fifth semester of their three-year course, whereas the simulation sessions take place toward the start of their fifth semester. Although this does not guarantee that every student will have had exactly the same clinical experience in the practice component of their course, they should at least have acquired similar basic skills and knowledge. So as not to disadvantage students from the control group and any other student from the same cohort, they are also invited to take part in the simulation training, but only after the second OSCE.

\section{RESULTS}

As the time course of an experiment of this nature is ongoing the authors are keen to report the results from the first two cohorts that have completed the study. A total of 101 students have taken part in the study. Unfortunately 34 of those students (33.7\%) withdrew from the study after the first OSCE session either because the sessions were organised in their own time $(18.8 \%)$, or they were allocated to the experimental group and failed to take part in the simulation sessions (14.9\%). Although students in this latter case attended the second OSCE session, their results were not considered 
for the study as they self selected themselves to join the control group. The results presented in Figure 2 include data from 67 candidates who have attended all the sessions required (66.3\%). This includes 38 students from the control group and 29 students from the experimental group. Although students have been randomly attributed to the control or experimental group, the mix of gender and abilities has been evenly distributed. The two groups obtained very similar scores for the first OSCE (Figure 2). This shows that students from both groups had a similar level of competence at the start of the study. The average age, the percentage of students with previous experience and the mean duration of this experience seem to favour the control group (Table 3). Statistical analysis of the results shows that the two groups have respectively improved their score by $6.76 \%$ and $13.43 \%$ for the second OSCE. This supports the conclusion that simulation training has enabled students from the experimental group to improve their skills and knowledge to a greater extent than those from the control group. The difference in improvement between the two groups is $6.67 \%$ in favour of the experimental group. An independent sample T-test of the individual students' OSCE scores shows that the difference in improvement between the two groups is highly significant $(p<0.05)$.

Figure 2

Table 3 
Recent research on the relation of medical students' experience, confidence and performance showed that there was no clear evidence that they could be related (Morgan \& Cleave-Hogg 2002). Graham and Scollon (2002) also concluded that improvement in the training of advanced life support skills did not lead to improved confidence. Similarly, results of the BHF project confidence questionnaire distributed to both groups of students immediately before the second OSCE session are very similar and do not enable us to determine whether or not the use of simulation leads to a higher level of confidence (Table 3).

\section{DISCUSSION}

Whether or not to include simulation in an undergraduate nursing curriculum requires careful considerations with respect to the financial and physical feasibility, and the possible benefits to students. The simulation technology investigated in this project allows for the acquisition of technical and non-technical skills that students will hopefully be able to transfer to their future clinical environment. It is essential to evaluate critically how effective is the use of realistic simulation in undergraduate nursing education. This research project will hopefully provide an unbiased answer to the latter issue. To date the results prove to be positive and the feedback from students is also very encouraging. Although there is no perceived difference in the level of confidence or perception of stress between the two groups of students, the limited period of exposure to simulation had a significant effect on the 
performance of the students. It has enabled students from the experimental group to improve their OSCE performance by an additional $6.67 \%$ over the students from the control group. The fact that students from the control group are on average older and had more experience in healthcare than students from the experimental group (Table 2) may have biased the difference in improvement and the confidence and stress level measures between the two groups. Continuing this study with further cohorts of students should reduce the differences and therefore any bias.

\section{CONCLUSION}

The current results support the use of simulation in undergraduate nursing education. However a very important point needs to be considered: a good tool is only as good if it is well used. Rystedt and Lindström (2001) suggest that the integration and design of the simulation have a great influence on what students can learn from it. This issue is further emphasised by Streufert et al. (2001) who advance that simulation design is a significant factor in its inferiority or superiority over other training methods. Thus the trainer or facilitator's teaching and training skills, and the simulation course are of great significance in what can be learnt and remembered during and after a simulation session. Simulation must form part of the learning

environment and be used appropriately to ensure effective learning (Kneebone 2003). In addition to the cost, another major barrier to the adoption of simulation technology is the lack of trainers experienced in using it (Ziv et 
al. 2000). It is expected that the results of this study will help and support other institutions which are in the process of purchasing simulation equipment. Hopefully it will also influence the design of future nursing curricula inside and outside the University to incorporate such teaching tools and training methods. Finally, as good as the simulation experience can be, it can not entirely replace some of the traditional teaching methods. Students will still need to learn at the bedside with real patients (Lane et al. 2001, Hegarty \& Bloch 2002).

\section{ACKNOWLEDGEMENTS}

This research program is supported by a grant from the British Heart Foundation (BHF Project number: Edcomm/Oct98/9d). The authors would like to acknowledge the valued support of the many lecturers from several departments of the University who have been involved as OSCE examiners, and especially Colin Harwood, Sharon Edwards, Guy Dean, Jay Ragoo, as well as to all the diploma nursing students who have taken part in the study. We also would like to thank Laerdal Medical Ltd (UK) for lending us a SimMan Universal Patient Simulator for the duration of the project.

\section{REFERENCES}


Alinier G 2003 Nursing students' and lecturers' perspectives of OSCE, incorporating simulation. Nurse Education Today 23/6 pp. 419-426. doi:10.1016/S0260-6917(03)00044-3

Alinier G, Hunt B, Gordon R HICESC: A University based simulation environment for Healthcare students. Annual Scientific meeting of the Society of Europeans for Simulation Applied to Medicine (SESAM). April 3rd -5th 2003, London, UK

Cioffi J 2001 Clinical simulations: development and validation. Nurse Education Today 21:477-486. doi:10.1054/nedt.2001.0584

Cleave-Hogg D, Morgan P 2002 Experiential learning in an anaesthesia simulation centre: analysis of students' comments. Medical Teacher 24(1):2326

Graham C, Scollon D 2002 Cardiopulmonary resuscitation training for undergraduate medical students: a five-year study. Medical Education 36:296298

Harden R, Gleeson F 1979 Assessment of clinical competence using Objective Structured Clinical Examination (OSCE). Medical Education 13:4154 
Hegarty M K, Bloch M B 2002 The use of simulators in intensive care training.

Current Anaesthesia and Critical Care 13:194-200

Issenberg S B, McGaghie W C, Hart I R, Mayer J W, Felner J M, Petrusa E R, Waugh R A, Brown D D, Safford R R, Gessner I H, Gordon D L, Ewy GA 1999 Simulation technology for health care professional skills training and assessment. Journal of the American Medical Association 282(9):860-866

Kneebone R 2003 Simulation in surgical training: educational issues and practical implications. Medical Education 37:267-277

Kohn L T, Corrigan J M, Donalson M S 1999 To Err is Human: Building a Safer Health System. National Academy Press, Washington DC.

Kolb D 1984 Experiential learning: experience as the source of learning and development. Prentice Hall, Englewood Cliffs.

Lane J L, Slavin S, Ziv A 2001 Simulation in medical education: A review. Simulation \& Gaming 32(3):297-314

Lave J, Wenger E 1991 Situated learning: Legitimate peripheral participation. Cambridge Press, Cambridge.

Mclndoe A 1999 Leading edge technology for anaesthesia training - medical simulation. Anaesthesia Care 3:4-8 
Morgan P, Cleave-Hogg D 2002 Comparison between medical students' experience, confidence and competence. Medical Education 36:534-539

Murray W B, Good M L, Gravenstein J S, Van Oostrom J H, Brasfield W G 2002 Learning about new anesthetic using a model driven, full human simulator. Journal of Clinical Monitoring and Computing 17:293-300

Niehaus A, DaRosa D, Markwell S, Folse R 1996 Is Test Security a concern when OSCE Stations are Repeated across Clerkship Rotations? Academic Medicine 71(3):287-289

Roberts I, Allsop P, Dickinson M, Curry P, Eastwick-Field P, Eyre G 1997 Airway management training using the laryngeal mask airway: a comparison of two different training programmes. Resuscitation 33:211-214

Rystedt H, Lindström B 2001 Introducing simulation technologies in nurse education: a nursing practice perspective. Nurse Education in Practice 1:134141. doi:10.1054/nepr.2001.0022

Streufert S, Satish U, Barach P 2001 Improving medical care: The use of simulation technology. Simulation \& Gaming 32(2):164-174

Thiagarajan S 1998 The myths and realities of simulations in performance technology. Educational Technology 38(5):35-41 
Treadwell I Grobler S 2001 Students' perceptions on skills training in simulation. Medical teacher 23(5):476-482

Ziv A, Small S D, Wolpe P R 2000 Patient safety and simulation-based medical education. Medical teacher 22(5):489-495 


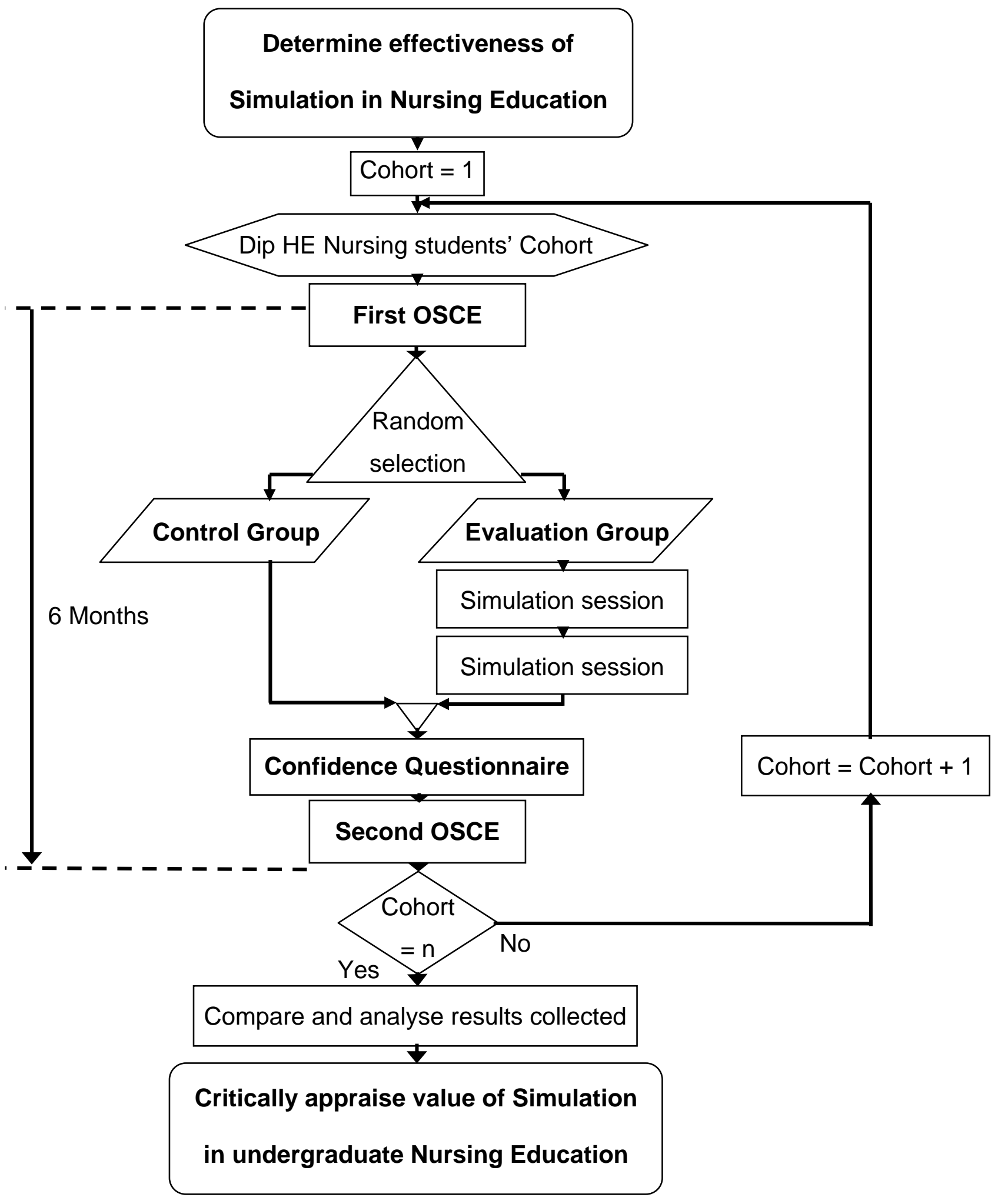

Figure 1: Flowchart diagram representing the study design which is repeated over several cohorts of diploma nursing students to determine the impact of the simulation training on their competence and confidence. 
List of OSCE stations:

\begin{tabular}{|c|c|c|}
\hline Stations & & Type \\
\hline ECG Electrodes positioning (3-Lead) & 1 & Practical \\
\hline Outcomes of incorrect ECG electrodes positioning & 2 & Theoretical \\
\hline dysrhythmia recognition (5 Rhythms) & 3 & Practical \\
\hline Kontron Monitor: Determining state of alarm settings & 4 & Practical \\
\hline HP Monitor: Modifying heart rate and temperature alarm settings & 5 & Practical \\
\hline Airway management (Oropharyngeal airway, bag ventilation) & 6 & Practical \\
\hline Safety aspects of the use of a defibrillator & 7 & Theoretical \\
\hline Pulse oximetry measurement (Finger \& ear probes) & 8 & Practical \\
\hline Electrical equipment set up problem & 9 & Theoretical \\
\hline Set up Volumetric infusion pump & 10 & Practical \\
\hline Determining the cause for syringe driver alarm & 11 & Practical \\
\hline Ventilator tubing installation & 12 & Practical \\
\hline Blood pressure measurement & 13 & Practical \\
\hline Electric bed positioning with entangled giving set & 14 & Practical \\
\hline Cardiac arrest signs & 15 & Theoretical \\
\hline
\end{tabular}

Table 1: List of OSCE stations used for the study. It is only for the practical stations that students are observed by an examiner. The marking of the theoretical stations is done after the session. 


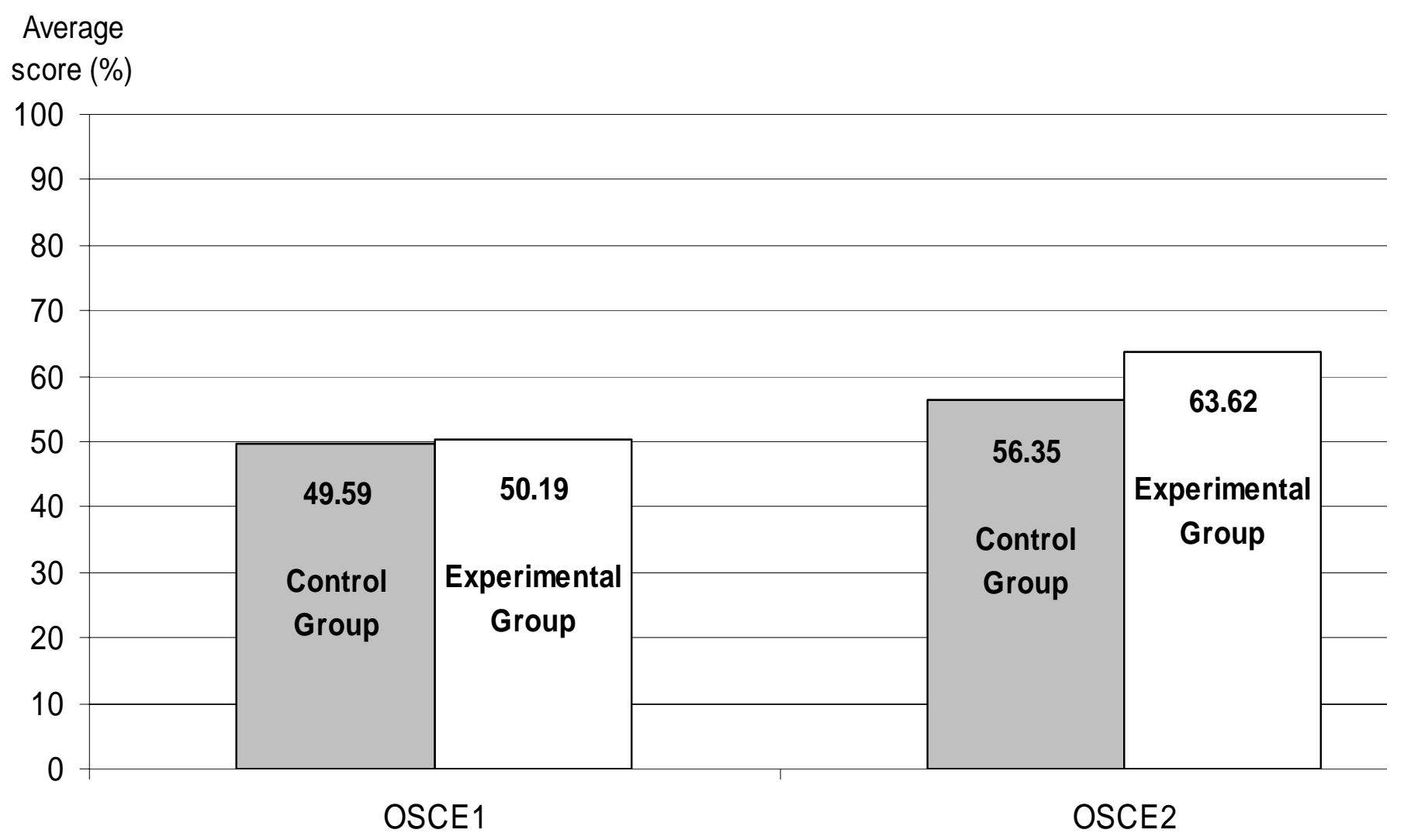

Figure 2: Bar chart representation of the average performance of the students from the control and experimental groups for the two OSCEs. 
Simulation session programme

\begin{tabular}{|c|c|c|c|}
\hline Duration & \multicolumn{3}{|c|}{ Programme } \\
\hline $10 \mathrm{~min}$ & \multicolumn{3}{|c|}{ Registration and Introduction } \\
\hline $20 \mathrm{~min}$ & \multicolumn{3}{|c|}{ Teamwork \& Communication discussion } \\
\hline $20 \mathrm{~min}$ & \multicolumn{3}{|c|}{ Introduction to Simulation and Familiarisation of students with SimMan } \\
\hline $5 \mathrm{~min}$ & \multicolumn{3}{|c|}{ Break } \\
\hline \multirow{4}{*}{$40 \min$} & \multicolumn{2}{|c|}{ Team 1} & Team 2 \\
\hline & Pair A & Pair B & Pair C and D \\
\hline & 1 Scenario & Observe simulation & \\
\hline & Observe simulation & 1 Scenario & Observe simulation \\
\hline $20 \min$ & \multicolumn{3}{|c|}{ Debriefing and feedback with comments from observers } \\
\hline $5 \mathrm{~min}$ & \multicolumn{3}{|c|}{ Break } \\
\hline \multirow{4}{*}{$40 \min$} & \multicolumn{2}{|c|}{ Team 1} & Team 2 \\
\hline & Pair A & Pair B & Pair C and D \\
\hline & 1 Scenario & Observe simulation & \\
\hline & Observe simulation & 1 Scenario & Observe simulation \\
\hline $20 \min$ & \multicolumn{3}{|c|}{ Debriefing and feedback with comments from observers } \\
\hline
\end{tabular}

Table 2: Plan of the 3-hour simulation session organised for two teams of four students from the experimental group. Students from team 1 were observers during the previous session, and students from team 2 will take part in the scenarios during the next simulation session. This enables to involve a maximum number of students and to maximise their exposure to simulation. 
Information concerning the students of the Experimental and Control groups

\begin{tabular}{l|c|c} 
& Experimental & Control \\
& Group & Group \\
\hline Number of students (n) & $29(43.3 \%)$ & $38(56.7 \%)$ \\
\hline Gender: Male / Female & $20.70 \% / 79.30 \%$ & $21.10 \% / 78.90 \%$ \\
\hline Average age (Years) & 29.41 & 33.18 \\
\hline Candidates with previous experience & $8(27.60 \%)$ & $17(44.70 \%)$ \\
\hline Average experience in years & 2.01 & 3.51 \\
\hline Confidence in working in a technological environment & & 3.50 \\
(1=very confident, 5=not confident at all) & 3.48 & 2.92 \\
\hline Stressfulness of working in a technological environment & & \\
(1=not stressful at all, 5=very stressful) & &
\end{tabular}

Table 3: Information concerning the major characteristics of the two study groups, and results of their confidence questionnaire. The statistics of this table are only representative of the students who validated their participation by attending all the sessions required. 